\title{
Overtube-assisted enteroscopy and capsule endoscopy for the diagnosis of small-bowel polyps and tumors: a systematic review and meta-analysis
}

Authors

Institutions
Marianny Sulbaran ${ }^{1}$, Eduardo de Moura' ${ }^{1}$ Wanderley Bernardo ${ }^{1}$, Cintia Morais', Joel Oliveira', Leonardo Bustamante-Lopez ${ }^{2}$, Paulo Sakai ${ }^{1}$, Klaus Mönkemüller $^{3}$, Adriana Safatle-Ribeiro ${ }^{1}$

Institutions are listed at the end of article. submitted 14. July 2015 accepted after revision 19. October 2015

\section{Bibliography}

Dol http://dx.doi.org/ 10.1055/s-0041-108261 Published online: 11.1.2016 Endoscopy International Open 2016; 04: E151-E163

(c) Georg Thieme Verlag KG Stuttgart · New York E-ISSN 2196-9736

\section{Corresponding author} Marianny Sulbaran, MD Rua Jose Antonio Coelho 207, Apartment 13 04011060 Sao Paulo Brazil Phone: (5511) 948526841 Fax: +55-11-31494790 mariannysulbaran@gmail.com
Background and study aims: Several studies have evaluated the utility of double-balloon enteroscopy (DBE) and capsule endoscopy (CE) for patients with small-bowel disease showing inconsistent results. The aim of this study was to determine the sensitivity and specificity of overtube-assisted enteroscopy (OAE) as well as the diagnostic concordance between OAE and CE for small-bowel polyps and tumors.

Patients and methods: We conducted a systematic review and meta-analysis of studies in which the results of OAE were compared with the results of CE for the evaluation of small-bowel polyps and tumors. When data for surgically resected lesions were available, the histopathological results of $\mathrm{OAE}$ and surgical specimens were compared. The sensitivity, specificity, positive likelihood ratio, and negative likelihood ratio for the diagnosis of small-bowel polyps and tumors were analyzed. Secondarily, the rates of diagnostic concordance and discordance between OAE and CE were calculated.

Results: There were 15 full-length studies with a total of 821 patients that met the inclusion crite-

\section{Introduction}

$\nabla$

About $5 \%$ of gastrointestinal neoplasms and $1 \%$ to $2 \%$ of gastrointestinal malignant tumors initially develop in the small bowel $[1,2]$. Small-bowel tumors have been difficult to diagnose as a consequence of their nonspecific presentation and the poor accessibility of the distal small bowel. Furthermore, many of these tumors can remain clinically silent for years [3].

Since the introduction of capsule endoscopy (CE) and overtube-assisted enteroscopy (OAE), the number of small-bowel polyps and tumors that are diagnosed has increased [4-6]. Obscure gastrointestinal bleeding (OGIB) is the main indication for using these enteroscopic modalities [610]. Importantly, the development of both dou- ria. The pooled sensitivity, specificity, positive likelihood ratio, and negative likelihood ratio were as follows: 0.89 (95\% confidence interval [CI] 0.84-0.93), with heterogeneity $X^{2}=41.23$ $(P=0.0002)$ and inconsistency $\left(I^{2}\right)=66.0 \% ; 0.97$ $(95 \%$ CI $0.95-0.98)$, with heterogeneity $X^{2}=45.27$ $(P=0.07)$ and inconsistency $\left(I^{2}\right)=69.1 \% ; 16.61$ (95\%CI 3.74-73.82), with heterogeneity Cochrane's $Q=225.19(P<0.01)$ and inconsistency $\left(I^{2}\right)=93.8 \%$; and 0.14 (95\%CI $\left.0.05-0.35\right)$, with heterogeneity Cochrane's $\mathrm{Q}=81.01(P<.01)$ and inconsistency $\left(I^{2}\right)=82.7 \%$, respectively. A summary receiver operating characteristic curve (SROC) curve was constructed, and the area under the curve (AUC) was 0.97 .

Conclusion: OAE is an accurate test for the detection of small-bowel polyps and tumors. OAE and $\mathrm{CE}$ have a high diagnostic concordance rate for small-bowel polyps and tumors.

This study was registered in the PROSPERO international database (www.crd.york.ac.uk/prospero/) with the study number CRD42015016000.

ble-balloon enteroscopy (DBE) $[11,12]$ and single-balloon enteroscopy (SBE) $[13,14]$ has made it possible to perform diagnostic and therapeutic procedures during a single examination. More recently, spiral enteroscopy (SE) has been introduced as an alternative to balloon-assisted enteroscopy for deep intubation of the small intestine [15].

Several studies have evaluated the utility of DBE and $C E$ in the evaluation of patients with suspected small-intestinal disease, including OGIB. However, the studies have shown inconsistent results and are largely limited by their small sample size [16]. Furthermore, these meta-analyses did not focus on small-bowel polyps and tumors. To the best of our knowledge, no systematic review has 
yet been conducted to evaluate $\mathrm{OAE}$ and $\mathrm{CE}$ for the diagnosis of small-bowel polyps and tumors.

The aim of this study was to determine the sensitivity and specificity of OAE, as well as the concordance rate between OAE and CE for the diagnosis of small-bowel polyps and tumors in patients presenting mainly with OGIB.

\section{Methods \\ $\nabla$}

This study was structured according to the PRISMA (Preferred Reporting Items for Systematic reviews and Meta-Analyses) recommendations [17]. It was registered in the PROSPERO international database (www.crd.york.ac.uk/prospero/) [18] with the study number CRD42015016000.

\section{Eligibility criteria}

Types of studies: We included comparative studies in which OAE (including DBE, SBE, and SE) and CE were performed to diagnose small-bowel disease in patients with OGIB. Our search was applied to all databases through November 2014. Studies were excluded if they did not report specific information on the results of $\mathrm{OAE}$ and $\mathrm{CE}$, included fewer than five patients, or were case reports, abstracts, or review articles. Studies that potentially shared enrolled patients were also excluded.

Participants: The patients in this meta-analysis had been given a diagnosis of OGIB, gastrointestinal polyposis, anemia, chronic abdominal pain, diarrhea, or suspected mass.

Interventions: We used studies that compared OAE, including $\mathrm{DBE}, \mathrm{SBE}$, and SE, with CE.

Outcome measures: True positives, true negatives, false positives, and false negatives were carefully extracted from the included studies. The primary outcome measures were sensitivity, specificity, positive likelihood ratio (PLR), and negative likelihood ratio (NLR) of OAE and the rates of concordance and discordance between $\mathrm{OAE}$ and $\mathrm{CE}$ for the diagnosis of small-bowel polyps and tumors.

\section{Information sources}

Three investigators (M.S., W.B., and K.M) independently performed a search of the medical literature up to November 2014. The following electronic databases were searched: MEDLINE (via PubMed), EMbase (Excerpta Medica database), LILACS (Latin American and Caribbean Health Science Literature), and Cochrane (via BVS [Biblioteca Virtual en Salud]). A manual search was also conducted of additional sources of information, such as bibliographies of identified articles, abstracts, and congress books. Potentially relevant articles were retrieved, and their reference lists were reviewed to identify studies that the search strategy might have missed. Papers were restricted to "full text."

\section{Search}

The following search terms were used for MEDLINE:

(Capsule Endoscopy OR Enteroscopy OR Double-Balloon Enteroscopy OR Double Balloon Enteroscopy OR Double-Balloon Enteroscopies OR Enteroscopies, Double-Balloon OR Enteroscopy, Double-Balloon OR Push-and-Pull Enteroscopy OR Enteroscopies, Push-and-Pull OR Enteroscopy, Push-and-Pull OR Push and Pull Enteroscopy OR Push-and-Pull Enteroscopies OR Single-Balloon Enteroscopy OR Spiral Enteroscopy) AND (small intestines OR small intestine OR small bowel OR duodenum OR intestines, small OR jejunum OR ileum) AND (angioectasia OR angiectasia
OR angiodysplasias OR arteriovenous malformation OR malformation, arteriovenous OR malformations, arteriovenous OR Dieulafoy OR ectasia, vascular OR bleeding OR gastrointestinal bleeding OR polyps OR polyposis OR tumors).

A similar strategy was used for the LILACS, EMbase, and Cochrane databases:

(Capsule OR Enteroscopy) AND (small intestine) AND (gastrointestinal bleeding OR polyps OR tumors).

\section{Studies selection}

Three investigators (M.S., W.B., and K.M) independently reviewed abstracts identified in the initial search to determine whether they were eligible for inclusion in a full-article review. The full papers were reviewed if there was disagreement about their inclusion. The study selection process was summarized through an adapted PRISMA flow diagram.

\section{Data collection process}

We extracted data from the included studies with a diagnostic studies checklist. The relevant data were then extracted from each study with a standardized extraction form. One review author extracted the data, and a second author checked the extracted data. Disagreements were resolved by discussion among the three review authors.

\section{Data items}

We extracted the following information from the trials:

- The study design, number of patients who underwent OAE and $\mathrm{CE}$, ages of the patients, years of patient enrollment, and main indications for interventions.

- The type of intervention, as different OAE modalities were considered (DBE, SBE, SE) as well as different models of CE. Regarding OAE, the insertion route, mean procedure time, mean depth of insertion, rate of complete examinations, and rate of complications were described. CE mean recording duration, rate of complete examinations, and rate of complications were described as well.

- The type of outcome measures, including true positives, true negatives, false positives, and false negatives. Based on these data, the primary outcome measures that underwent meta-analysis were sensitivity and specificity of OAE. The rates of diagnostic concordance and discordance between OAE and CE were described as a systematic review only. When data for surgically resected specimens were available, OAE biopsy results were compared with the final surgical histopathological diagnosis.

The sensitivity and specificity of OAE were calculated by establishing $\mathrm{CE}$ as the reference test for the diagnosis of small-bowel pathology. This was because of its ability to visualize the entire small bowel in a higher proportion of patients compared with OAE [19-21].

\section{Risk for bias in individual studies}

Two reviewers worked independently with adequate reliability to measure the risk for bias and validate study eligibility. The data were assessed with QUADAS-2 (Quality Assessment of Diagnostic Accuracy Studies [22] to evaluate the risk for bias and applicability in the study.

\section{Summary measures}

The primary outcome measures were sensitivity, specificity, PLR, and NLR, as well as the pretest probability of OAE for the diagno- 


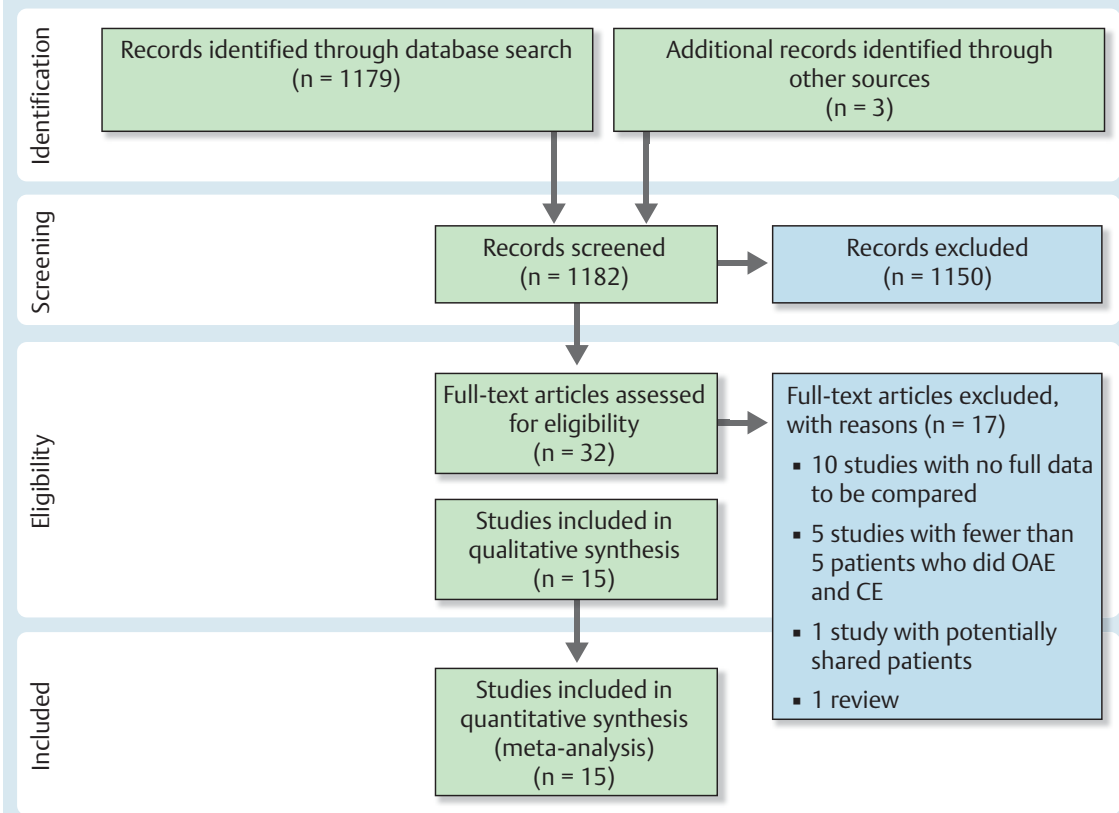

Fig. 1 Flow diagram of the process for selecting eligible studies. OAE, overtube-assisted enteroscopy; CE, capsule endoscopy.

sis of small-bowel polyps and tumors. The rates of concordance and discordance between OAE and CE for the diagnosis of smallbowel polyps and tumors were also assessed.

\section{Planned methods of analysis}

Based on a comparison of OAE and CE results, $2 \times 2$ statistical tables were constructed for each study. Where 0 counts occurred in at least 1 cell of study data, a continuity correction of 0.5 was added to every value for that study to make the calculation of sensitivity and specificity. Based on these data, the sensitivity, specificity, PLR, and NLR (with corresponding 95\% confidence intervals [CIs]) of enteroscopy were calculated. Pooled results with corresponding $95 \%$ CIs were derived by using the random effects model. A summary receiver operating characteristic curve (SROC) was constructed based on the Moses-Shapiro-Littenberg method [23]. The area under the curve (AUC) was computed as a measure of the overall performance of enteroscopy to accurately differentiate patients with small-bowel polyps and tumors from those without. A preferred test has an AUC close to 1, and a poor test has an AUC close to 0.5. Cochrane's Q2 test was used to assess heterogeneity, and the $I^{2}$ statistic was used to measure inconsistency A value of $I^{2}$ below $30 \%$ was not considered to be statistically significant. $I^{2}$ values of $30 \%$ to $60 \%$ were considered to represent moderate heterogeneity, between $50 \%$ and $90 \%$ substantial heterogeneity, and between $75 \%$ and $100 \%$ considerable heterogeneity [24]. The analysis was performed with Meta-DiSc version 1.4 statistical software (Unit of Clinical Biostatistics team of the Ramon y Cajal Hospital, Madrid, Spain). The Critically Appraised Topic (CAT) software was also used to calculate pretest probability values.

\section{Risk for bias within studies}

To evaluate the risk for bias within the studies, QUADAS-2 was applied to each of the studies. According to QUADAS-2, bias was classified as related to patient selection and/or OAE and/or CE and/or flow and timing. Studies of high quality were defined as those with low risk answers to at least three of four key items. Studies of poor quality were those that failed or had an unclear answer to three of the four items. Moderate quality was assigned for every other possibility.

\section{Additional analysis}

The rates of concordance and discordance per patient between OAE and CE for the diagnosis of small-bowel polyps and tumors were assessed.

\section{Results \\ $\nabla$}

The initial search yielded 1182 citations, and 1150 articles were excluded after preliminary abstract review. There were 32 fulllength articles reviewed for eligibility. Detailed reasons for exclusion are outlined in Appendix A. A total of 15 full-length studies met the inclusion criteria ( $\bullet$ Fig. 1).

\section{Study characteristics}

A total of 821 patients underwent both OAE and CE for a variety of indications, including OGIB, gastrointestinal polyposis, anemia, chronic abdominal pain, diarrhea, and suspected mass. The main characteristics of the included studies are detailed in 0 Table 1. Enrolled studies were published between 2005 and 2014. Eight studies were prospectively designed, and seven were retrospective. Five studies were from Japan [19,20,25 -27] three from the United States [28-30], two from Italy [21,31], and one from each of the following countries: Germany [32], Romania [33], Korea [34], Canada [35], and China [36]. Four studies were conducted at multiple centers: two from Italy, one from Korea, and one from the United States. DBE was the OAE approach used in 11 studies, whereas SBE was used in three studies and SE in two studies. The M2A PillCam (Given Imaging, Yoqneam, Israel) was used in nine studies. The capsule model used in six of the studies was not specified. Data on the sequence of examinations, time between tests, $\mathrm{OAE}$ mean procedure time, $\mathrm{CE}$ recording duration, OAE depth of insertion, and percentages of OAE and CE complete examinations and complications were extracted. 


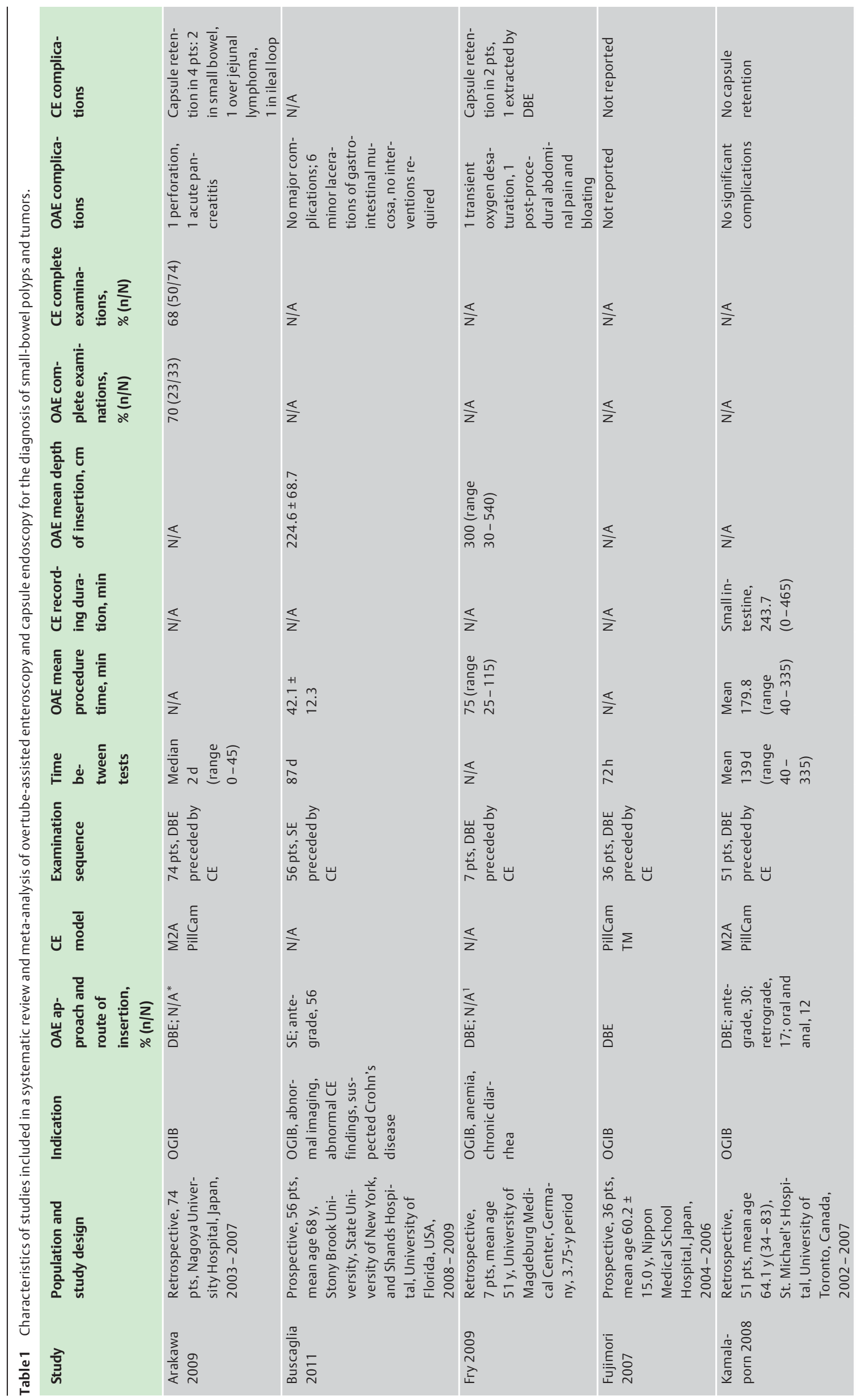




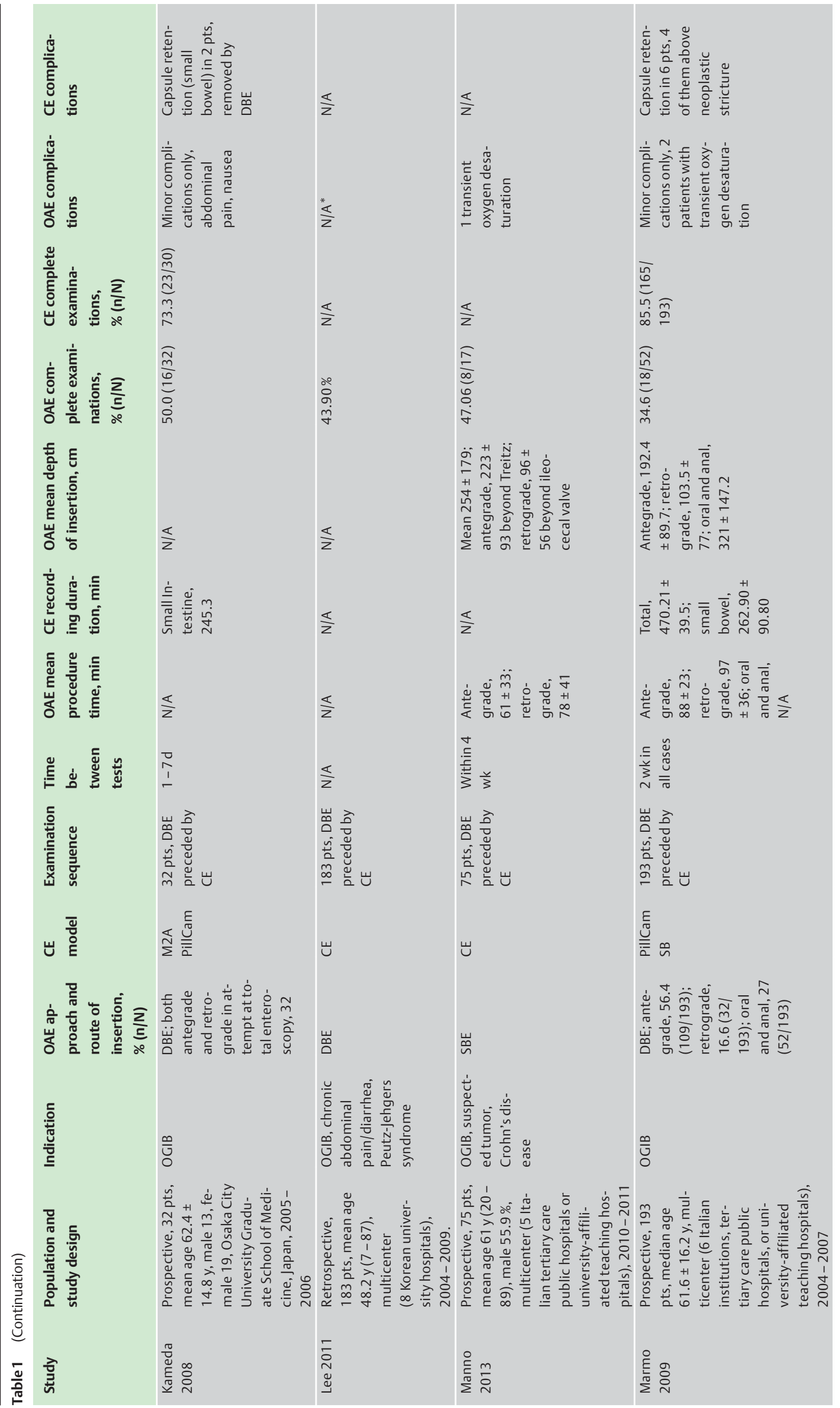



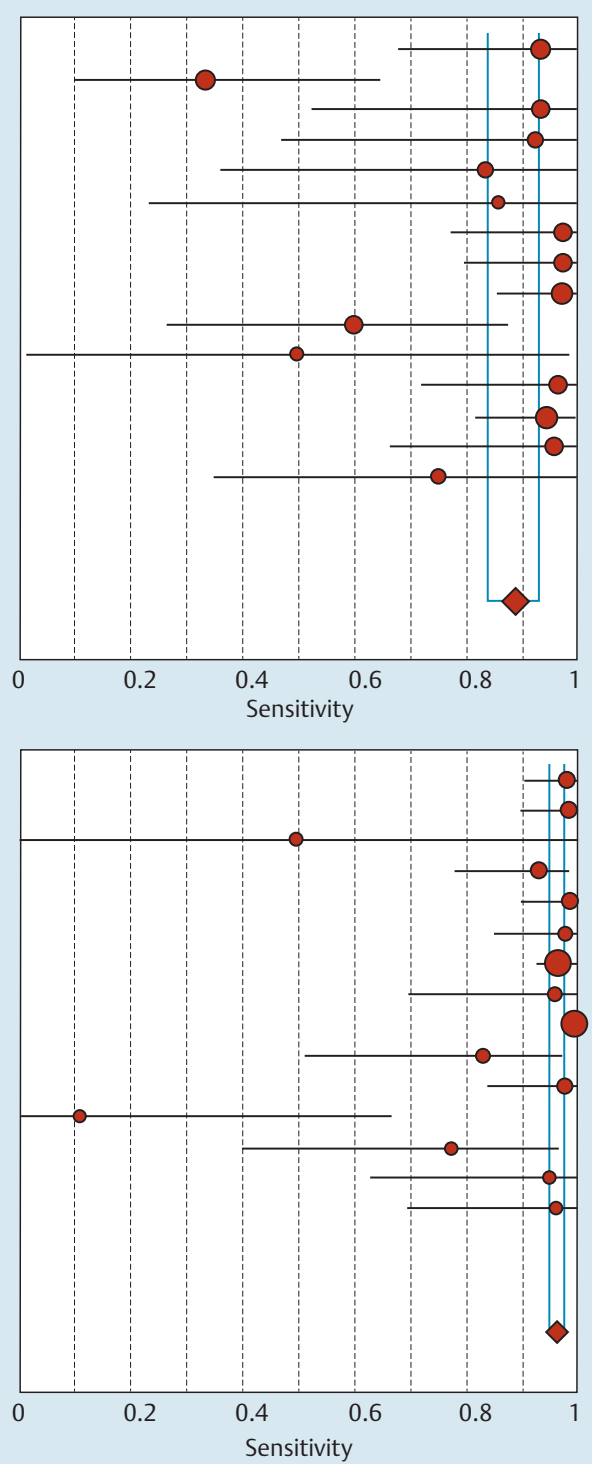

Arakawa 2008

Buscaglia 2011

Fry 2008

Fujimori 2007

Kamalapom 2008

Kameda 2008

Lee 2011

Manno 2013

Marmo 2009

Matsumoto 2005

Nakamura 2006

Partridge 2011

Sethi 2014

Vere 2009

Xiao-bo 2007

Pooled Sensitivity $=0.89$ (0.84 to 0.93 )

Chi-square $=41.23 ; \mathrm{df}=14(P=0.0002)$ Inconsistency (I-square) $=66.0 \%$

Arakawa 2008

Buscaglia 2011

Fry 2008

Fujimori 2007

Kamalapom 2008

Kameda 2008

Lee 2011

Manno 2013

Marmo 2009

Matsumoto 2005

Nakamura 2006

Partridge 2011

Sethi 2014

Vere 2009

Xiao-bo 2007

Pooled Sensitivity $=0.97$ (0.95 to 0.98$)$

Chi-square $=45.27 ; \mathrm{df}=14(P=0.0000)$

Inconsistency $(\mathrm{l}$-square $)=69.1 \%$
Sensitivity (95\% Cl)

$0.98 \quad(0.91-0.65)$

$0.99 \quad(0.90-1.00)$

$0.50 \quad(0.00-1.00)$

$0.93 \quad(0.78-0.99)$

$0.99 \quad(0.90-1.00)$

$0.98 \quad(0.85-1.00)$

$0.97 \quad(0.93-0.99)$

$0.95 \quad(0.70-1.00)$

$1.00 \quad(0.97-1.00)$

$0.83 \quad(0.52-0.98)$

$0.98 \quad(0.84-1.00)$

$0.11 \quad(0.00-0.67)$

$0.78 \quad(0.40-0.97)$

$0.95 \quad(0.63-1.00)$

$0.796(0.70-1.00)$
Fig. 2 Forest plots of overtube-assisted enteroscopy pooled sensitivity and specificity for diagnosis of small bowel polyps and tumors. $\mathrm{Cl}$, confidence interval.

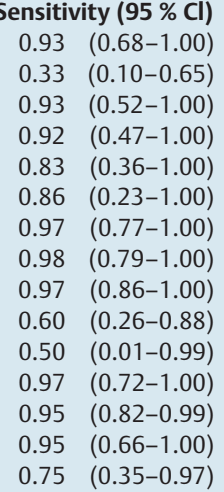



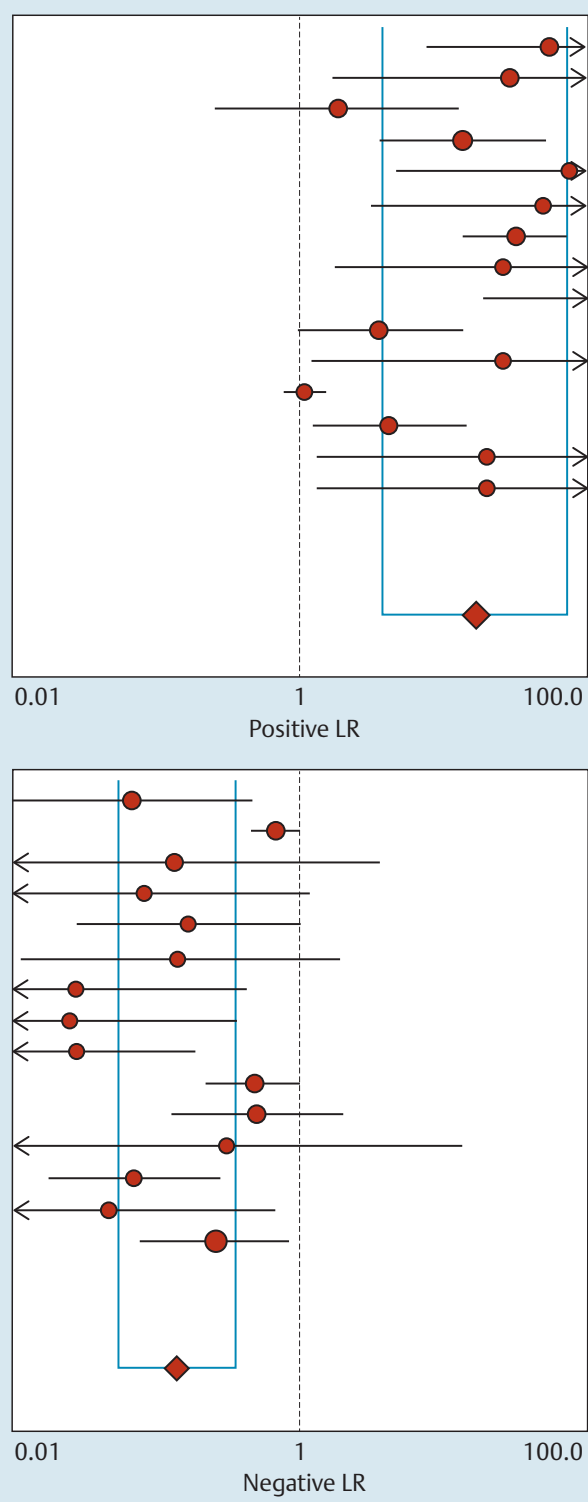

Arakawa 2008

Buscaglia 2011 Fry 2008

Fujimori 2007

Kamalapom 2008

Kameda 2008

Lee 2011

Manno 2013

Marmo 2009

Matsumoto 2005

Nakamura 2006

Partridge 2011

Sethi 2014

Vere 2009

Xiao-bo 2007

Random Effects Model

Pooled Positive LR $=16.61$ (3.74 to 73.82)

Cochran- $\mathrm{Q}=225, \mathrm{df}=14(P=0.0000)$

Inconsistency (I-square) $=93.8 \%$

Tau-squared $=7,4326$
Positive LR (95 \% Cl)

$(7.85-385.27)$

$29.67 \quad(1.68-523.23)$

$1.87 \quad(0.26-13.38)$

$13.85 \quad(3.56-53.80)$

$75.83 \quad(4.71-1221.96)$

$50.57 \quad(3.13-816.23)$

$32.11 \quad(12.50-76.37)$

$26.34 \quad(1.73-400.22)$

304.54 (19.12-4849.88)

$3.60 \quad(0.92-14.05)$

$26.50 \quad(1.22-574.01)$

$(0.77-1.53)$

$4.26 \quad(1.25-14.49)$

$20.09 \quad(1.34-301.24)$

$20.25 \quad(1.30-316.53)$
Fig. 3 Forest plots of OAE pooled positive and negative likelihood ratios for diagnosis of small bowel polyps and tumors. LR, likelihood ratio; $\mathrm{Cl}$, confidence interval.

\begin{tabular}{llr} 
& \multicolumn{2}{l}{ Negative LR (95 \% Cl) } \\
Arakawa 2008 & 0.07 & $(0.01-0.45)$ \\
Buscaglia 2011 & 0.67 & $(0.45-1.01)$ \\
Fry 2008 & 0.13 & $(0.00-3.68)$ \\
Fujimori 2007 & 0.03 & $(0.01-1.18)$ \\
Kamalapom 2008 & 0.17 & $(0.03-1.01)$ \\
Kameda 2008 & 0.15 & $(0.01-1.89)$ \\
Lee 2011 & 0.03 & $(0.00-0.43)$ \\
Manno 2013 & 0.03 & $(0.00-0.39)$ \\
Marmo 2009 & 0.03 & $(0.00-0.19)$ \\
Matsumoto 2005 & 0.48 & $(0.22-1.07)$ \\
Nakamura 2006 & 0.51 & $(0.13-2.04)$ \\
Partridge 2011 & 0.31 & $(0.01-13.52)$ \\
Sethi 2014 & 0.07 & $(0.02-0.28)$ \\
Vere 2009 & 0.05 & $(0.00-0.69)$ \\
Xiao-bo 2007 & 0.25 & $(0.03-0.87)$
\end{tabular}

Random Effects Model

Pooled Negative LR $=0.14$ (0.05 to 0.35 )

Cochran- $\mathrm{Q}=81.01, \mathrm{df}=14(P=0.0000)$

Inconsistency (l-square) $=82.7 \%$

Tau-squared $=2,3471$

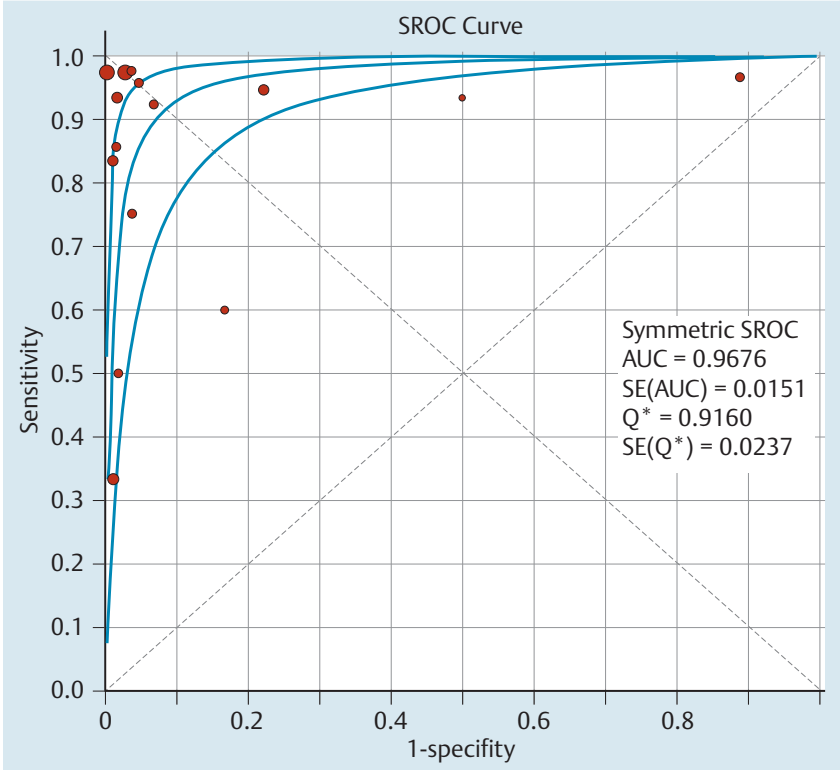

Fig. 4 Summary receiver operating characteristics (SROC) curve for the diagnosis of small-bowel polyps and tumors. AUC, area under the curve; Q*, Q index; SE, standard error. majority of studies has primarily limited previous analyses. In this study, 821 patients were included. Thus, it was possible to analyze and compare CE and OAE findings. We found that OAE is an accurate test for the detection of small-bowel polyps and tumors in patients presenting mainly with OGIB. OAE and CE have a high concordance rate for the diagnosis of small-bowel polyps and tumors.

Even though we did not aim to compare different enteroscopy approaches, the sensitivity and specificity of SBE were similar to pooled OAE sensitivity and specificity. The overall diagnostic yield for SBE was higher than in previously published studies [30]. The improved diagnostic yield may be related to the routine use of CE before SBE in these cases. Although there are no data on the specific increase in diagnostic yield in the diagnosis of smallbowel polyps and tumors for CE and SBE vs SBE alone, it is reasonable to propose that $\mathrm{CE}$ and OAE should be used in a combined approach for the diagnosis of small-bowel polyps and tumors.

Of note, the sensitivity of SE was lower than the overall OAE sensitivity. Some explanations for this observation were suggested. The first is that rotational advancement enteroscopy is simply not as effective as balloon-assisted enteroscopy for deep intes- 


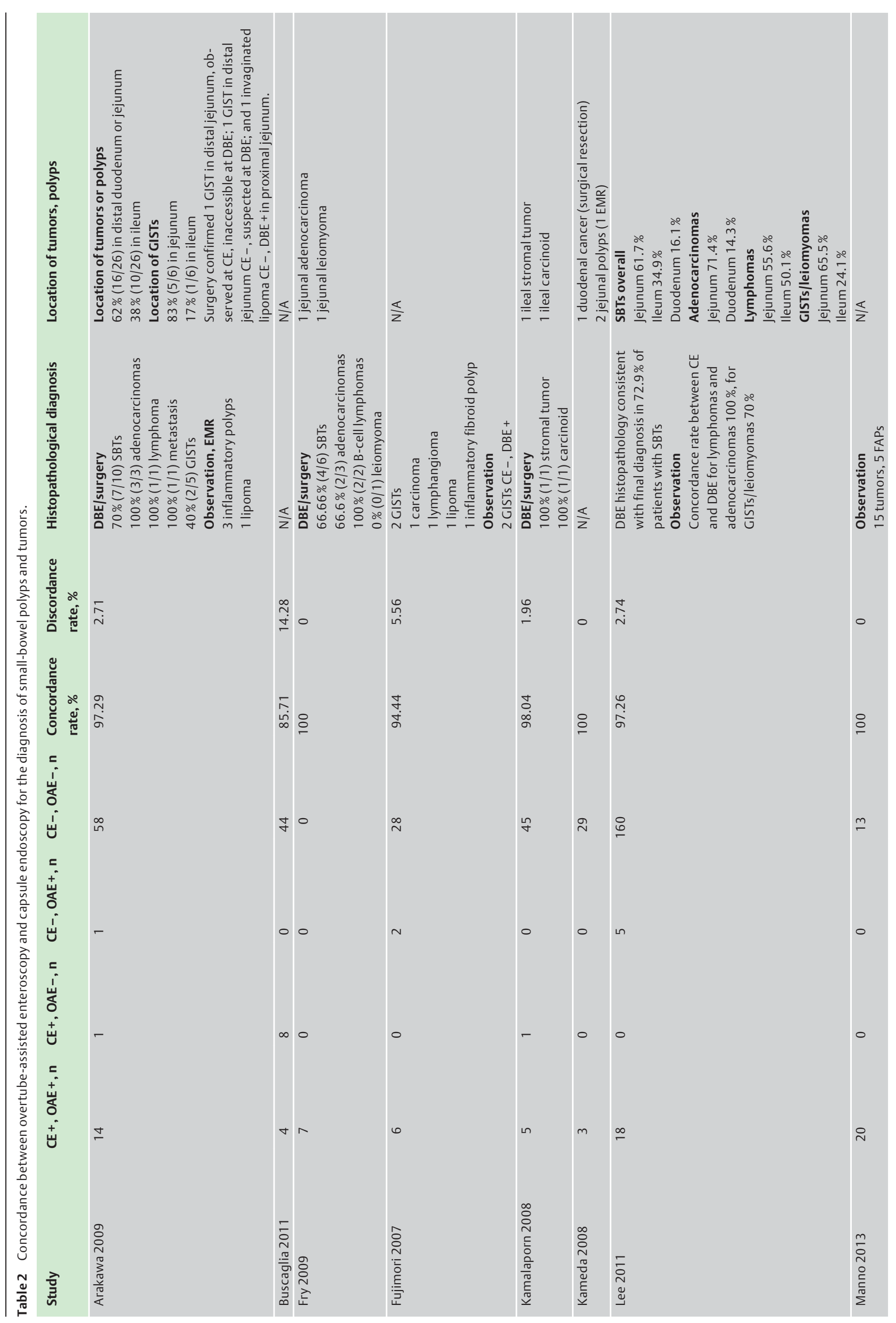




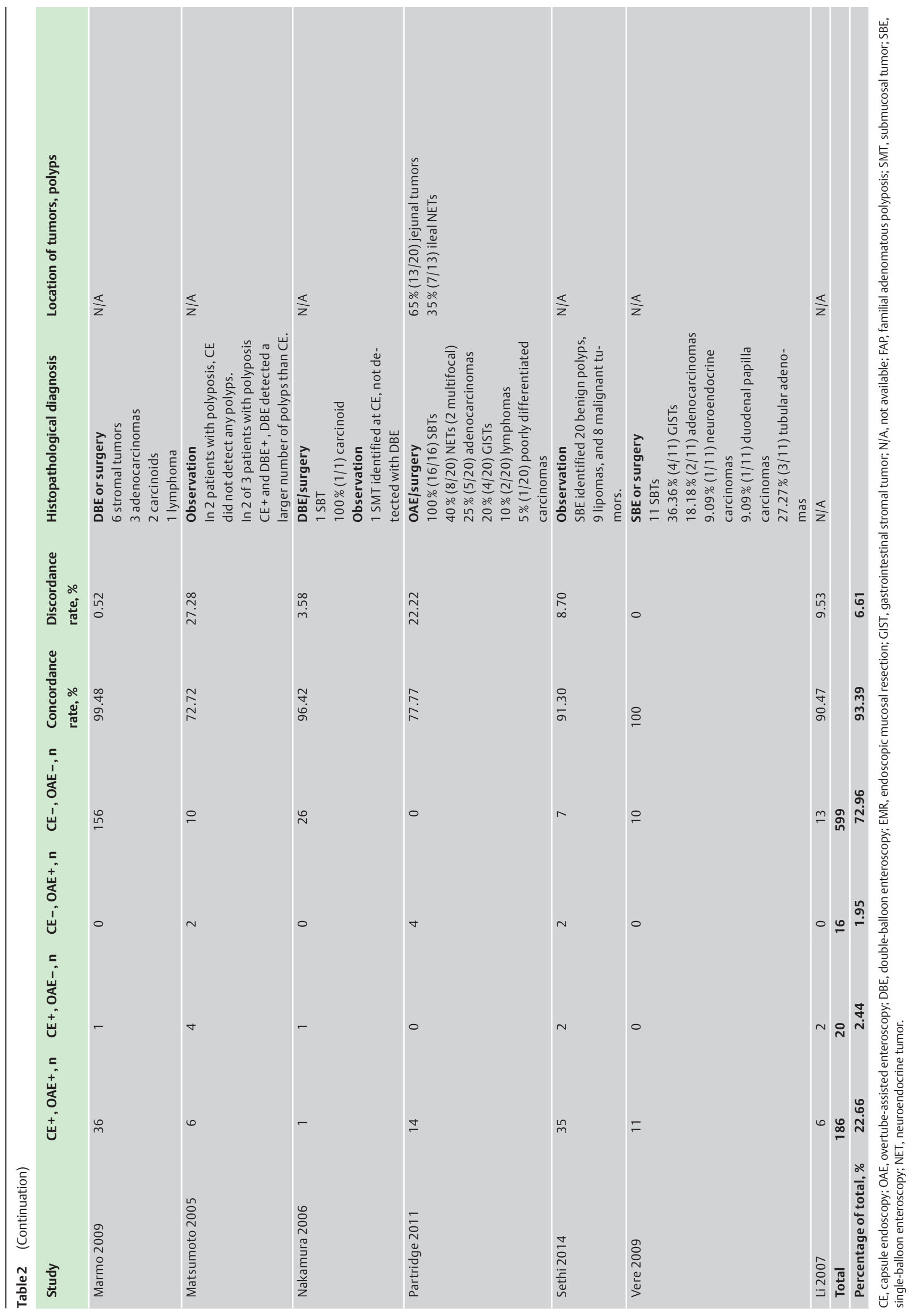




\begin{tabular}{|llllll|}
\hline & Selection of patients & OAE & CE & Time and flow & Study quality \\
\hline Arakawa 2009 & L & U & L & L & Good \\
\hline Buscaglia 2011 & L & H & L & H & Moderate \\
\hline Fry 2009 & H & H & L & L & Moderate \\
\hline Fujimori 2007 & L & H & L & L & Good \\
\hline Kamalaporn 2008 & L & H & L & H & Moderate \\
\hline Kameda 2008 & L & L & L & U & Good \\
\hline Lee 2011 & H & H & L & U & Poor \\
\hline Manno 2013 & L & H & L & L & Good \\
\hline Marmo 2009 & L & L & L & L & Good \\
\hline Matsumoto 2005 & H & L & L & L & Good \\
\hline Nakamura 2006 & L & L & L & L & Good \\
\hline Partridge 2011 & L & H & L & U & Moderate \\
\hline Sethi 2014 & L & H & L & L & Good \\
\hline Vere 2009 & L & U & L & U & Moderate \\
\hline Li 2007 & L & H & L & L & Good \\
\hline
\end{tabular}

Table 3 Risk for bias according to QUADAS-2 (Quality Assessment of Diagnostic Accuracy Studies).

OAE, overtube-assisted enteroscopy; CE, capsule endoscopy; L, low risk for bias; $\mathrm{U}$, unclear risk for bias; $\mathrm{H}$, high risk for bias.

tinal intubation. In addition, small polyps may be overlooked during enteroscopy and can also be overdiagnosed by CE [28]. Future perspectives on enteroscopy modalities should also be taken into consideration. The NaviAid AB (Advancing Balloon) device (SMART Medical Systems, Ra'anana, Israel) is a balloon-assisted enteroscopy device that uses a through-the-scope balloon to allow deep intubation of the small intestine with the use of a standard colonoscope. This system has a short learning curve and can be used without the assistance of another endoscopist [37]. More studies are required in order to evaluate its diagnostic yield compared with CE findings.

Neither CE nor OAE is an independent gold standard test for the diagnosis of small-bowel polyps and tumors. However, in our study, CE was defined as the reference test for sensitivity and specificity extraction data because it has provided a higher number of complete examinations of the small intestine compared with OAE. In this systematic review, the CE complete examination rates ranged from $68 \%$ to $90.9 \%$, whereas the OAE complete examination rates ranged from $16.7 \%$ to $70 \%$, in concordance with previous data $[15,30,32,34]$. Additionally, in a large European multicenter trial that included 5129 patients who underwent $\mathrm{CE}$, confirmation of the diagnosis and location of the lesion was obtained in all cases by conventional endoscopic or surgical means. In that study, an agreement rate of $92.8 \%$ between $\mathrm{CE}$ and the final endoscopic or surgical diagnosis for single lesions was demonstrated [38].

Although there was a $93.39 \%$ concordance rate between OAE and $\mathrm{CE}$, both tests can miss lesions. There were 20 cases detected by capsule and missed by OAE, and 16 cases missed by capsule and seen on OAE. Some possible explanations have been suggested because each method has its own strengths and limitations, and especially when small-bowel tumors and polyps are evaluated, these tests should be considered complementary

Total enteroscopy cannot always be achieved by OAE [6,32]. A combination of antegrade and retrograde DBE approaches with the intention of total enteroscopy was successful in $85.7 \%$ of patients [6]. Furthermore, OAE is intrinsically an invasive method of enteroscopy, and not all patients with a negative evaluation on the first procedure are willing to undergo a second one via the complementary route. It is therefore likely that a complete examination of the small bowel still cannot be achieved with OAE in certain patients. Additionally, although a greater number of polyps were detected with DBE than with $\mathrm{CE}$, in the area explored by DBE in patients with polyposis, the majority of the polyps or tumors detected only by CE were located in the portion of the small bowel that was not evaluated by DBE [27]. These facts suggest that even for the surveillance of patients with polyposis syndromes, in whom the therapeutic impact of OAE is greater, CE and OAE show complementary benefits [32].

Taking into consideration studies that provided surgical specimen histopathological data $[32,39,40]$, we demonstrated that in $80 \%$ of the DBE biopsies that missed the diagnosis, the lesions were of subepithelial origin. The misdiagnosed lesions included three gastrointestinal stromal tumors (GISTs), one leiomyoma, and one adenocarcinoma, demonstrating a low histological diagnostic yield for DBE in subepithelial tumors. This is in concordance with the findings of others [3], who also explained that the initially sampled tissue can be superficial to the targeted lesion. Additionally, $100 \%$ agreement regarding histological and laparoscopically resected specimens was shown for DBE [39], with the fact taken into consideration that in five cases of suspected GIST, biopsies were not obtained. Afterward, four GISTs and one gastrointestinal autonomic tumor (GANT) were further confirmed surgically. Chen et al. also analyzed the reasons for diagnoses missed by DBE in a large series of 440 patients who underwent $D B E$, in which the 11 patients with negative $D B E$ results did indeed have tumors detected through surgery or CE. In this group of DBE false negatives, the reasons for the missed diagnoses were inadequate depth of insertion in $45 \%$ (5/11), suboptimal choice of insertion approach in 36\% (4/11), and the presence of tumors that had exophytic growth with normal intestinal mucosa in $18 \%(2 / 11)[41]$.

CE has its own assets and drawbacks as well. Mass lesions are typically focal and thus more likely to be missed. False-positive data for submucosal tumors on CE may also be due to transient bulges into the lumen of the small bowel [39]. Although it has been stated that $\mathrm{CE}$ can miss mass lesions located in the proximal small bowel because of the high velocity of the capsule as it passes along the duodenal sweep and the currently limited angle of view $[40,42,43]$, no clear association between tumor location and missed CE lesions was found in this review. Four CE-negative tumors were reported to be submucosal, three were GISTs, and one was an invaginated lipoma, findings concordant with those of a series of 150 consecutive CE examinations establishing that CE missed two small-bowel GISTs and one small-bowel mesenteric tumor diagnosed by surgery or previous contrast-enhanced computed tomography [42]. Five significant lesions missed by CE were found with other imaging modalities: DBE in three patients 
and computed tomographic enterography and magnetic resonance enterography in the remaining two patients, and four of these lesions were located in the proximal jejunum [43].

The capsule system that was mostly frequently used in the included studies was the M2A PillCam, so the capsule model did not influence the variability of our results. Current capsule limitations, such as reduced angle of view, may be overcome in the near future with the recently developed 360-degree panoramic viewing capsule. More studies would be needed to demonstrate significant differences between the diagnostic yield of the panoramic capsule and that of currently widely available CE systems [44]. In contrast, at the present time, OAE may provide clearer and more comprehensive images, aided by air insufflation in the small-bowel lumen, the possibility of removing debris from the mucosa with water, and the ability to push and pull the enteroscope to re-inspect a suspicious small-bowel segment.

Even though we did not aim to study the therapeutic yield of OAE, this approach offers therapeutic advantages that should be evaluated, especially for patients with polyposis syndromes. Whereas DBE was useful for the diagnosis of various types of polyps and tumors, its major endoscopic therapeutic impact was evident in patients with familial adenomatous polyposis syndrome or Peutz-Jeghers syndrome [32]. Another important issue is the higher overall prevalence of small-bowel polyps and tumors in the patients included in our study $(26.36 \% \pm 22.68)$ vs what has been previously reported by others $(5 \%-17.4 \%)[1,45]$. This may be explained by the fact that the patients included in our study were at high risk for polyps or tumors because they had been selected to undergo OAE, with or without an abnormal CE study, or had concerning symptoms, mainly OGIB. We presented the pretest probability for the subgroup of patients who underwent both tests in each study, which does not necessarily represent the prevalence of the total number of patients who underwent OAE. Additionally, studies that included patients with benign lesions or polyposis were not excluded. This fact also could have influenced our overall higher reported prevalence. Nevertheless, our study does reflect the patients undergoing OAE and CE in clinical practice.

Regarding complications, it is important to state that even though OAE is a more invasive technique than $\mathrm{CE}, 0.36 \%$ of the patients experienced significant complications (two cases of perforation and one of acute pancreatitis). Capsule retention occurred in $2 \%$ of the patients, and either OAE or surgical methods were used for retrieval.

Our study has potential limitations. First, and important, is the limited number of comparison data with a gold standard method, such as intraoperative enteroscopy or surgery, found in most of the included studies. However, intraoperative enteroscopy is now rarely performed. Second, although histological confirmation is required for choosing the most adequate therapeutic option, histological confirmation by OAE may sometimes guide therapeutics other than surgery, such as chemotherapy. This is especially important in cases of malignant lymphoma or metastasis. Third, most studies had a relatively small sample size, and heterogeneity may also have limited the study. Furthermore, most studies used CE as an initial test, and its results served as a guide for the OAE route of insertion and localization of lesions. Indeed, OAE was frequently performed with an unblinded CE result, which introduced a higher risk for bias. However, this approach reflects the current standard of care, and it would not make sense to randomize patients to undergo OAE first when CE is a less invasive test.
In summary, our data show that OAE is an accurate test for the detection of small-bowel polyps and tumors in patients presenting mainly with OGIB. In addition, OAE and CE have a high concordance rate for the diagnosis of small-bowel polyps and tumors. Nevertheless, the two tests may have both false-negative and false-positive results. Thus, a combined approach is suggested. CE may be used initially to guide the OAE route of insertion and optimize its therapeutic applications.

\section{Appendix A}

List of full-length reviewed articles excluded, with reasons for exclusion.
Studies that could not compare full data between capsule endoscopy and overtube-assisted enteroscopy
1. Ross et al. Dig Dis Sci 2008 [46]
2. Choi et al. Gastrointest Endosc 2007 [47]
3. Schäfer et al. Z Gastroenterol 2007 [48]
4. Cangemi et al. J Clin Gastroenterol 2013 [3]
5. Pérez-Cuadrado et al. Rev Esp Enferm Dig 2006 [49]
6. Almeida et al. Dig Dis Sci 2009 [50]
7. Riccioni et al. Surg Endosc 2012 [39]
8. Frantz et al. Gastrointest Endosc 2010 [51]

Case studies or papers that did not enroll at least five patients who underwent both capsule endoscopy and overtube-assisted enteroscopy

1. Zagorowicz et al. World J Gastroenterol 2013 [42]

2. Akamatsu et al. Dig Endosc 2010 [52]

3. Chong et al. Gastrointest Endosc 2006 [53]

4. Postgate et al. Gastrointest Endosc 2008 [43]

5. Rondonotti et al. Endoscopy 2008 [38]

\section{Studies from which data for polyps and tumors could not be extracted \\ 1. Chen et al. World J Gastroenterol 2013 [41] \\ 2. Albert et al. Eur J Gastroenterol Hepatol 2008 [45]}

\section{Studies that potentially shared enrolled patients with} other included studies

1. Honda et al. Gastrointest Endosc 2012 [40]

The studies of Honda et al. [40] and Arakawa et al. [25] potentially shared included patients. If one takes into consideration that patients included in the study of Arakawa et al. had OGIB, and patients in the study of Honda et al. all had small-bowel tumors, we decided to exclude the study of Honda et al. because super-estimated values of sensitivity and specificity could be computed.

\section{Review}

1. Alexander and Leighton. Curr Opin Gastroenterol 2009 [54]

\section{Competing interests: None}

\footnotetext{
Institutions

${ }^{1}$ Gastrointestinal Endoscopy Service. Gastroenterology Department, Clinics Hospital, University of Sao Paulo School of Medicine, Sao Paulo, Brazil

2 Surgical Division, Gastroenterology Department, Clinics Hospital, University of Sao Paulo School of Medicine, Sao Paulo, Brazil

${ }^{3}$ Basil I. Hirschowitz Endoscopic Center of Excellence, Division of Gastroenterology and Hepatology, UAB School of Medicine, Birmingham, Alabama, USA
} 


\section{References}

1 Gay G, Delvaux M. Small-bowel endoscopy. Endoscopy 2008; 40: 140 146

2 Rustgi AK. Small intestinal neoplasms. In: Feldman M, Friedman LS, Brandt LI (eds.) Sleisenger and Fordtran's gastrointestinal and liver disease. $8^{\text {th }}$ ed. New York, NY: Elsevier; 2006: 2703-2712

3 Cangemi DJ, Patel MK, Gomez V et al. Small bowel tumors discovered during double-balloon enteroscopy: analysis of a large prospectively collected single-center database. J Clin Gastroenterol 2013; 47: 769 772

4 Moglia A, Menciassi A, Dario $P$ et al. Clinical update: endoscopy for small-bowel tumours. Lancet 2007; 370: 114-116

5 Kala Z, Válek $V$, Kysela $P$ et al. A shift in the diagnostics of the small intestine tumors. Eur J Radiol 2007; 62: 160-165

6 Yamamoto H, Kita H, Sunada K et al. Clinical outcomes of double-balloon endoscopy for the diagnosis and treatment of small-intestinal diseases. Clin Gastroenterol Hepatol 2004; 2: 1010-1016

7 Kandulski A, Fry LC, Malfertheiner P. Detection of jejunal carcinoid in a patient with obscure-overt gastrointestinal bleeding by double-balloon enteroscopy. Clin Gastroenterol Hepatol 2008; 6: A26

8 Iddan G, Meron G, Glukhovsky A et al. Wireless capsule endoscopy. Nature 2000; 405: 417

9 Meron GD. The development of the swallowable video capsule (M2A). Gastrointest Endosc 2000; 52: 817 -819

10 Ginsberg GG, Barkun AN, Bosco JJ et al. Wireless capsule endoscopy: August 2002. Gastrointest Endosc 2002; 56: 621-624

11 Yamamoto H, Sekine $Y$, Sato $Y$ et al. Total enteroscopy with a nonsurgical steerable double-balloon method. Gastrointest Endosc 2001; 53: 216- 220

12 Di Caro S, May A, Heine DG et al. The European experience with doubleballoon enteroscopy: indications, methodology, safety, and clinical impact. Gastrointest Endosc 2005; 62: 545 - 550

13 Tsujikawa T, Saitoh Y, Andoh A et al. Novel single-balloon enteroscopy for diagnosis and treatment of the small intestine: preliminary experiences. Endoscopy 2008; 40: $11-15$

14 Hartmann D, Eickhoff A, Tamm $R$ et al. Balloon-assisted enteroscopy using a single-balloon technique. Endoscopy 2007; 39: E276

15 Akerman PA, Agrawal D, Cantero D et al. Spiral enteroscopy with the new DSB overtube: a novel technique for deep peroral small-bowel intubation. Endoscopy 2008; 40: 974-978

16 Pasha SF, Leighton JA, Das A et al. Double-balloon enteroscopy and capsule endoscopy have comparable diagnostic yield in small-bowel disease: a meta-analysis. Clin Gastroenterol Hepatol 2008; 6: 671-676

17 Liberati A, Altman DG, Tetzlaff J et al. The PRISMA statement for reporting systematic reviews and meta-analyses of studies that evaluate health care interventions: explanation and elaboration. J Clin Epidemiol 2009; 62: e1-e34

18 National Institute for Health Research. Welcome to PROSPERO: international prospective register of systematic reviews. Available from: http://www.crd.york.ac.uk/prospero/ (Accessed 20 October 2015)

19 Nakamura $M$, Niwa $Y$, Ohmiya $N$ et al. Preliminary comparison of capsule endoscopy and double-balloon enteroscopy in patients with suspected small-bowel bleeding. Endoscopy 2006; 38: 59-66

20 Kameda $N$, Higuchi $K$, Shiba $M$ et al. A prospective, single-blind trial comparing wireless capsule endoscopy and double-balloon enteroscopy in patients with obscure gastrointestinal bleeding. J Gastroenterol 2008; 43: 434-440

21 Marmo R, Rotondano G, Casetti T et al. Degree of concordance between double-balloon enteroscopy and capsule endoscopy in obscure gastrointestinal bleeding: a multicenter study. Endoscopy 2009; 41: 587 592

22 Whiting PF, Rutjes AW, Westwood ME et al. QUADAS-2: a revised tool for the quality assessment of diagnostic accuracy studies. Ann Intern Med 2011; 155: 529-536

23 Moses LE, Shapiro D, Littenberg B. Combining independent studies of a diagnostic test into a summary ROC curve: data-analytic approaches and some additional considerations. Stat Med 1993; 12: 1293-1316

24 Higgins JP, Thompson SG. Quantifying heterogeneity in a meta-analysis. Stat Med 2002; 21: 1539-1558

25 Arakawa $D$, Ohmiya $N$, Nakamura $M$ et al. Outcome after enteroscopy for patients with obscure GI bleeding: diagnostic comparison between double-balloon endoscopy and videocapsule endoscopy. Gastrointest Endosc 2009; 69: 866-874

26 Fujimori S, Seo T, Gudis K et al. Diagnosis and treatment of obscure gastrointestinal bleeding using combined capsule endoscopy and double balloon endoscopy: 1-year follow-up study. Endoscopy 2007; 39 : $1053-1058$

27 Matsumoto T, Esaki M, Moriyama T et al. Comparison of capsule endoscopy and enteroscopy with the double-balloon method in patients with obscure bleeding and polyposis. Endoscopy 2005; 37: 827-832

28 Buscaglia JM, Richards R, Wilkinson MN et al. Diagnostic yield of spiral enteroscopy when performed for the evaluation of abnormal capsule endoscopy findings. J Clin Gastroenterol 2011; 45: 342-346

29 Partridge BJ, Tokar JL, Haluszka 0 et al. Small bowel cancers diagnosed by device-assisted enteroscopy at a U.S. referral center: a five-year experience. Dig Dis Sci 2011; 56: 2701 - 2705

30 Sethi S, Cohen J, Thaker AM et al. Prior capsule endoscopy improves the diagnostic and therapeutic yield of single-balloon enteroscopy. Dig Dis Sci 2014; 59: 2497-2502

31 Manno M, Riccioni ME, Cannizzaro $R$ et al. Diagnostic and therapeutic yield of single balloon enteroscopy in patients with suspected smallbowel disease: results of the Italian multicentre study. Dig Liver Dis 2013; 45: $211-215$

32 Fry LC, Neumann H, Kuester D et al. Small bowel polyps and tumours: endoscopic detection and treatment by double-balloon enteroscopy. Aliment Pharmacol Ther 2009; 29: 135-142

33 Vere CC, Foarfă C, Streba CT et al. Videocapsule endoscopy and single balloon enteroscopy: novel diagnostic techniques in small bowel pathology. Rom J Morphol Embryol 2009; 50: 467-474

34 Lee BI, Choi H, Choi KY et al. Clinical characteristics of small bowel tumors diagnosed by double-balloon endoscopy: KASID multi-center study. Dig Dis Sci 2011; 56: 2920-2927

35 Kamalaporn $P$, Cho $S$, Basset $N$ et al. Double-balloon enteroscopy following capsule endoscopy in the management of obscure gastrointestinal bleeding: outcome of a combined approach. Can J Gastroenterol 2008; 22: $491-495$

$36 \mathrm{Li} \mathrm{XB}, \mathrm{Ge} \mathrm{ZZ,} \mathrm{Dai} \mathrm{J} \mathrm{et} \mathrm{al.} \mathrm{The} \mathrm{role} \mathrm{of} \mathrm{capsule} \mathrm{endoscopy} \mathrm{combined} \mathrm{with}$ double-balloon enteroscopy in diagnosis of small bowel diseases. Chin Med J (Engl) 2007; 120: 30-35

37 Ali $R$, Wild $D$, Shieh F et al. Deep enteroscopy with a conventional colonoscope: initial multicenter study by using a through-the-scope balloon catheter system. Gastrointest Endosc 2015; 82: 855-860

38 Rondonotti E, Pennazio $M$, Toth $E$ et al. Small-bowel neoplasms in patients undergoing video capsule endoscopy: a multicenter European study. Endoscopy 2008; 40: 488-495

39 Riccioni ME, Cianci R, Urgesi R et al. Advance in diagnosis and treatment of small bowel tumors: a single-center report. Surg Endosc 2012; 26: $438-441$

40 Honda $W$, Ohmiya $N$, Hirooka $Y$ et al. Enteroscopic and radiologic diagnoses, treatment, and prognoses of small-bowel tumors. Gastrointest Endosc 2012; 76: $344-354$

41 Chen WG, Shan GD, Zhang $H$ et al. Double-balloon enteroscopy in small bowel tumors: a Chinese single-center study. World J Gastroenterol 2013; 19: 3665 - 3671

42 Zagorowicz ES, Pietrzak AM, Wronska E et al. Small bowel tumors detected and missed during capsule endoscopy: single center experience. World J Gastroenterol 2013; 19: 9043 -9048

43 Postgate A, Despott E, Burling D et al. Significant small-bowel lesions detected by alternative diagnostic modalities after negative capsule endoscopy. Gastrointest Endosc 2008; 68: 1209-1214

44 Friedrich $K$, Gehrke S, Stremmel W et al. First clinical trial of a newly developed capsule endoscope with panoramic side view for small bowel: a pilot study. J Gastroenterol Hepatol 2013; 28: 1496 - 1501

45 Albert JG, Schülbe R, Hahn L et al. Impact of capsule endoscopy on outcome in mid-intestinal bleeding: a multicentre cohort study in 285 patients. Eur J Gastroenterol Hepatol 2008; 20: 971 - 977

46 Ross A, Mehdizadeh S, Tokar J et al. Double balloon enteroscopy detects small bowel mass lesions missed by capsule endoscopy. Dig Dis Sci 2008; 53: $2140-2143$

47 Choi H, Choi KY, Eun CS et al. Korean experience with double balloon endoscopy: Korean Association for the Study of Intestinal Diseases multi-center study. Gastrointest Endosc 2007; 66: S22 - S25

48 Schäfer C, Rothfuss K, Kreichgauer HP et al. Efficacy of double-balloon enteroscopy in the evaluation and treatment of bleeding and nonbleeding small bowel disease. Z Gastroenterol 2007; 45: 237-243

49 Pérez-Cuadrado E, Más P, Hallal H et al. Double-balloon enteroscopy: a descriptive study of 50 explorations. Rev Esp Enferm Dig 2006; 98: $73-81$ 
50 Almeida N, Figueiredo P, Lopes $S$ et al. Double-balloon enteroscopy and small bowel tumors: a South-European single-center experience. Dig Dis Sci 2009; 54: 1520-1524

51 Frantz DJ, Dellon ES, Grimm IS et al. Single-balloon enteroscopy: results from an initial experience at a US tertiary-care center. Gastrointest Endosc 2010; 72: $422-426$

52 Akamatsu T, Kaneko Y, Ota $\mathrm{H}$ et al. Usefulness of double balloon enteroscopy and video capsule endoscopy for the diagnosis and management of primary follicular lymphoma of the gastrointestinal tract in its early stages. Dig Endosc 2010; 22: 33 - 38

53 Chong AK, Chin BW, Meredith CG. Clinically significant small-bowel pathology identified by double-balloon enteroscopy but missed by capsule endoscopy. Gastrointest Endosc 2006; 64: 445 - 449

54 Alexander JA, Leighton JA. Capsule endoscopy and balloon-assisted endoscopy: competing or complementary technologies in the evaluation of small bowel disease? Curr Opin Gastroenterol 2009; 25: 433 437 